\title{
The association of chronic endometritis with mid-trimester loss due to cervical incompetence and the outcome of laparoscopic cervical cerclage
}

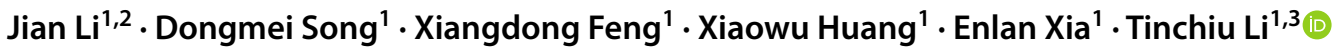

Received: 22 April 2020 / Accepted: 12 March 2021 / Published online: 3 April 2021

(c) The Author(s), under exclusive licence to Springer-Verlag GmbH Germany, part of Springer Nature 2021

\begin{abstract}
Purpose To examine the association of chronic endometritis (CE) with cervical incompetence (CI) in Chinese women with mid-trimester loss, and the impact of the presence of CE on the outcome of laparoscopic cervical cerclage (LCC).

Methods This retrospective cohort study included a study group of 293 women with mid-trimester loss due to CI (group I) and a comparison group of 332 women with recurrent first-trimester miscarriage (group II). Immunohistochemical study using CD138 epitope for the diagnosis of CE was completed in all subjects. Pre-conception LCC was undertaken in 247 women in the study group (group I). The study was approved by Institutional Review Board (IRB) (number 2015FXHEC-KY005). Results The prevalence of CE in group I was $42 \%$, significantly $(P<0.001)$ higher than that of $23.5 \%$ in group II. Among 247 women in group I, there were no significant difference in mid-trimester loss rate, preterm delivery rate and term delivery rate in women with and without $\mathrm{CE}(2.2,12.0,85.8 \%$ vs. $1.8,10.1,88.1 \%$ respectively) and between women with $\mathrm{CE}$ treated and not treated with antibiotics prior to conception $(2.3,9.3,88.4 \%$ vs. $2.0,14.3,83.7 \%$ respectively).

Conclusions Mid-trimester loss due to cervical incompetence is associated with chronic endometritis; However, the presence or not of $\mathrm{CE}$ and whether it was treated with antibiotics prior to conception did not appear to significantly influence the obstetric outcomes of women with CI after LCC.
\end{abstract}

Keywords Cervical incompetence $\cdot$ Chronic endometritis $\cdot$ Mid-trimester loss $\cdot$ Pre-conception laparoscopic cervical cerclage

\section{Introduction}

There are several recognized causes of mid-trimester loss, namely uterine malformations, cervical incompetence (CI), infections, fetal and placental anomalies, genetic abnormalities and thrombophilia [1-4]. Environmental co-exposures, such as alcohol consumption, tobacco smoke exposure and socio-economic status are related to recurrent first trimester

Tinchiu Li

tinchiu.li@gmail.com

1 Hysteroscopy Center, Fu Xing Hospital, Capital Medical University, Beijing, China

2 Center for Reproductive Medicine, Dalian Obstetrics and Gynecology Hospital, Dalian, China

3 Assisted Reproductive Technology Unit, Department of Obstetrics and Gynecology, Prince of Wales Hospital New Territories, Chinese University of Hong Kong, Hong Kong, China miscarriage, stillbirth or preterm delivery [5-8] and the risk of miscarriage and preterm delivery may be reduced by modification of these risk factors [7]. Among these underlying conditions, cervical incompetence is an important cause of recurrent spontaneous mid-trimester loss [3, 9]. It typically presents with painless cervical dilation; occasionally it may also present with rupture of membranes or uterine tightening especially if ascending infection has occurred following cervical dilatation. Recurrence mid-trimester loss rates were significantly lower after prophylactic vaginal or abdominal cerclage compared with no cerclage [9].

Chronic Endometritis (CE) is a persistent inflammation characterized by the presence of plasma cell in the endometrial stroma [10]. Recent studies have shown that the immunohistochemical staining for CD138, a cell surface proteoglycan that is expressed on plasma cells, improves the diagnostic accuracy of chronic endometritis [11]. CE is often asymptomatic and has been reported to contribute to recurrent miscarriage and recurrent implantation failure 
[12-14]. However, it is not known if chronic endometritis is also associated with cervical incompetence in women with mid-trimester loss.

In this study, we wish to examine the prevalence of CE among a cohort of women with mid-trimester loss associated with CI, compared to a cohort of women with recurrent first-trimester loss but without a history of mid-trimester loss, and to determine if the outcome of pre-conception laparoscopic cervical cerclage (LCC) is influenced by the presence of CE.

\section{Methods}

\section{Subjects}

This retrospective cohort study was conducted in the Hysteroscopy center, Fu Xing Hospital, Capital Medical University, Beijing, China. A total of 625 Chinese women who had a history of spontaneous mid-trimester loss or recurrent first trimester loss, were referred to the hospital for investigation and management between May 2015 and December 2017. Pregnancy outcomes of women who underwent preconception laparoscopic cervical cerclage were followed up until December 2019. The study was approved by Institutional Review Board (IRB) (number 2015FXHEC-KY005) and written consent was obtained from subjects participating in the study.

Women with spontaneous mid-trimester loss and a clinical diagnosis [15] of cervical incompetence belong to the main study group (group I, $n=293$ ), which is further divided into two sub-groups: (a) women who presented with a classical history of cervical incompetence characterized by painless cervical dilation (without uterine contractions or pain, or prolonged rupture of membrane) (group Ia, typical group, $n=198$ ); and (b) women with CI but presented with an atypical history characterized by either irregular uterine tightening or rupture of membranes $>24 \mathrm{~h}$ prior to delivery (group $\mathrm{Ib}$, atypical group, $n=95)$. Women with recurrent first trimester loss ( 2 or more clinical miscarriages $<14$ week) but without any history of mid-trimester loss constituted the comparison group (group II, $n=332$ ) for this study.

\section{Hysteroscopy and endometrial biopsy}

During the period of study, women who were referred to our unit because of recurrent first trimester loss or mid-trimester loss underwent routine hysteroscopic assessment and endometrial biopsy to rule out intra-uterine pathology.

Hysteroscopy was performed using a $4.5-\mathrm{mm} 30$ degree rigid hysteroscope (Olympus, Germany) as an out-patient procedure without the use of any anesthesia. Normal saline solution was used to distend the uterine cavity at $100 \mathrm{mmHg}$ pressure. An endometrial biopsy was routinely obtained after hysteroscopy with the use of a curette.

\section{CD138 staining for diagnosis of CE}

The paraformaldehyde-fixed paraffin-embedded endometrial sections were immune-stained with monoclonal antibody against epitope CD-138 at 1:100 dilution (Biocare Medical, Concord, CA) $[10,16]$. The immunoreactive cells were enumerated per 10 high power field (HPF) randomly chosen with non-overlapping stromal areas. The biopsies were graded as positive for chronic endometritis (CE) when there was one or more plasma cell identified per $10 \operatorname{HPF}[10,16]$. Regarding observer variability in diagnosis, two competent blinded histopathologists separately reviewed a set of 40 slides, the concordance of result in diagnosis of $\mathrm{CE}$ is shown in Table $1(K=0.881, P<0.001)$.

\section{Antibiotic therapy}

Between May 2015 and Nov 2016, CD138 staining was routinely performed along with traditional histological evaluation of endometrial biopsy specimens. During this period, 141 specimens from the study group were examined; subjects whose biopsy specimen showed CE received a course of antibiotics (500 mg levofloxacin (clinafloxacin), once a day and $500 \mathrm{mg}$ metronidazole twice a day) for 2 weeks.

From December 2016 onwards, CD-138 staining ceased to be part of routine histopathological examination and so the diagnosis of $\mathrm{CE}$ was no longer available to clinicians. There were 152 specimens in this study in which CD-138 staining was performed retrospectively in archived specimens. In this group of subjects, as the clinicians were not aware of the diagnosis of CE, antibiotic therapy was not administered.

\section{Laparoscopic cerclage}

Among women in group I, 247 subjects underwent preconception laparoscopic cervical cerclage (LCC). The primary indication for LCC was a clinical diagnosis of cervical incompetence resulting in one or more mid-trimester losses; the secondary indications included: (a) failure of a previous vaginal cerclage to achieve a live birth $(n=177)$;

Table 1 Inter-observer variability in the immunohistochemical diagnosis of CE

\begin{tabular}{llcl}
\hline First observer & \multicolumn{2}{l}{ Second observer } & Consistence \\
\cline { 2 - 3 } & Negative & Positive & \\
\hline Negative & 27 & 2 & $K=0.881$ \\
Positive & 0 & 11 & \\
\hline
\end{tabular}


(b) previous cervical trauma or surgery which rendered it difficult to perform transvaginal cerclage $(n=19)$; and (c) patient request for pre-conception cerclage $(n=51)$.

LCC was performed with the use of simplified laparoscopic cervical cerclage (SLCC) technique as previously described [17]. In brief, the straight needle was inserted into cervical wall 2-3 mm medial to the lateral edge of the cervix at the level of the cervical isthmic junction, without dissecting the bladder off the lower uterine segment and without separating the uterine vessels.

\section{Follow-up}

Clinical and laboratory data was retrieved from hospital record; additionally, telephone contact was made by JL to obtain various reproductive outcome data (211 conceptions confirmed by ultrasound) with a minimum follow-up period of 24 months after the surgery. A flow chart of patient participation and follow-up is shown in Fig. 1.

\section{Statistical analysis}

The various clinical and laboratory data were analyzed with the use of SPSS 21. The difference in demographic characteristics, prevalence of chronic endometritis and delivery and miscarriage rate between groups was analyzed with the use of Pearson Chi-square or Fisher exact tests. Student's $T$ test was used to compare quantitative data between groups. Statistical significance was defined as $P<0.05$. Where appropriate, Bonferroni's correction was applied, in which case the significance level between groups was adjusted to $P<0.0167$.

Fig. 1 Flow chart of the study

\section{Results}

\section{Demographics}

The demographics of the three groups of subjects (groups Ia, Ib and II) are summarized in Table 2. There was no difference in age, BMI and previous parity and miscarriage history among the three groups.

\section{Prevalence of chronic endometritis}

The prevalence of CE in group I (123/293, 42.0\%) was significantly $(P<0.001)$ higher than that of group II $(78 / 332$, $23.5 \%)$. $(\mathrm{OR}=2.36 ; 95 \% \mathrm{CI}=1.67-3.32)$. The difference remained significant after Bonferroni's correction (Fig. 2). Within group I, the prevalence of CE in subgroup Ib (48/95, $50.5 \%)$ was significantly $(P=0.040)$ higher than that in group Ia $(75 / 198,37.9)$. $(\mathrm{OR}=1.68 ; 95 \% \mathrm{CI}=1.02-2.75)$. The difference did not reach statistical significance after Bonferroni's correction (Fig. 2).

Within subgroup $\mathrm{Ib}$, the prevalence of $\mathrm{CE}$ in subjects who presented with prolonged ruptured membranes $(21 / 39$; $53.8 \%)$ was not significantly $(P=0.650)$ different to that of subjects who presented with tightening $(27 / 56 ; 48.2 \%)$. $(\mathrm{OR}=0.798 ; 95 \% \mathrm{CI}=0.352-1.810)$.

\section{Relationship between CE and number of miscarriages}

Within group I, the prevalence of CE in subjects with 1 , 2 and $\geq 3$ mid-trimester miscarriages were $38 / 70$ (54.3\%),

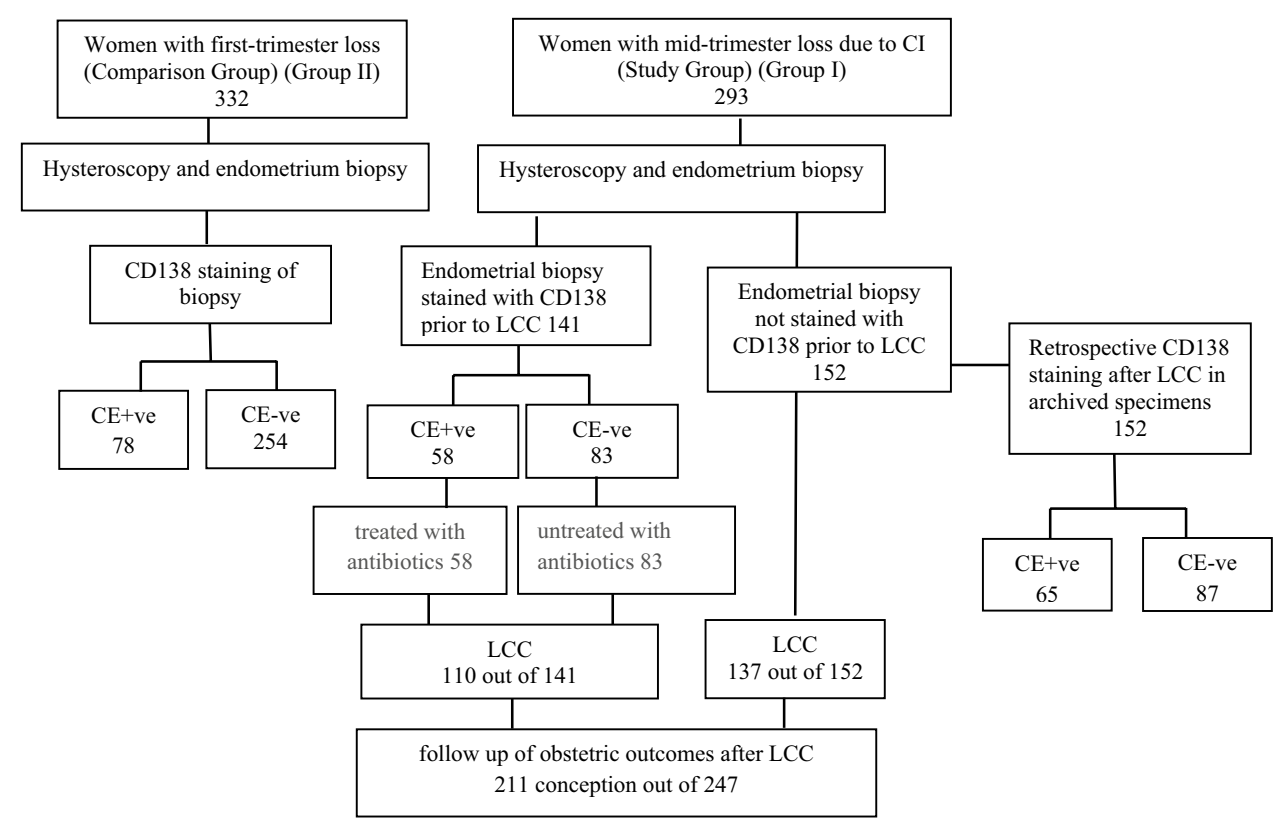


Table 2 Demographic details of subjects included in the study
Fig. 2 A comparison of the prevalence of chronic endometritis in three groups of subjects. Group Ia-women with a classical history of cervical incompetence. Group Ib-women with atypical history of cervical incompetence. Group II-Women with recurrent first trimester loss (the comparison group)

\begin{tabular}{lllll}
\hline & Group Ia $n=198$ & Group Ib $n=95$ & Group II $n=332$ & $P^{*}$ \\
\hline Maternal age $^{\text {a }}$ (years) & $32(21-42)$ & $31(25-43)$ & $30(23-41)$ & 0.637 \\
$\mathrm{BMI}^{\mathrm{a}},\left(\mathrm{kg} / \mathrm{m}^{2}\right)$ & $23.1(19.3-37.8)$ & $22.6(18.4-38.5)$ & $22.2(18.7-36.2)$ & 0.453 \\
Parity, $n$ & & & \\
0 & $178(89.9 \%)$ & $89(93.7 \%)$ & $314(94.6 \%)$ & 0.120 \\
$\geq 1$ & $20(10.1 \%)$ & $6(6.3 \%)$ & $18(5.4 \%)$ & \\
Previous mid-trimester miscarriage, $n$ & $2(1-8)$ & $2(1-5)$ & 0 & $0.550^{* *}$ \\
Previous first trimester miscarriage, $n$ & $0(0-4)$ & $0(0-8)$ & $2(2-6)$ & $0.536^{* *}$ \\
\hline
\end{tabular}

* $P$ value of the Chi-square test or Fisher's exact test for categorical variables and Student's $T$-test or the Mann-Whitney test for continuous variables

**Comparison between groups Ia and Ib only

${ }^{a}$ Median (range in brackets)

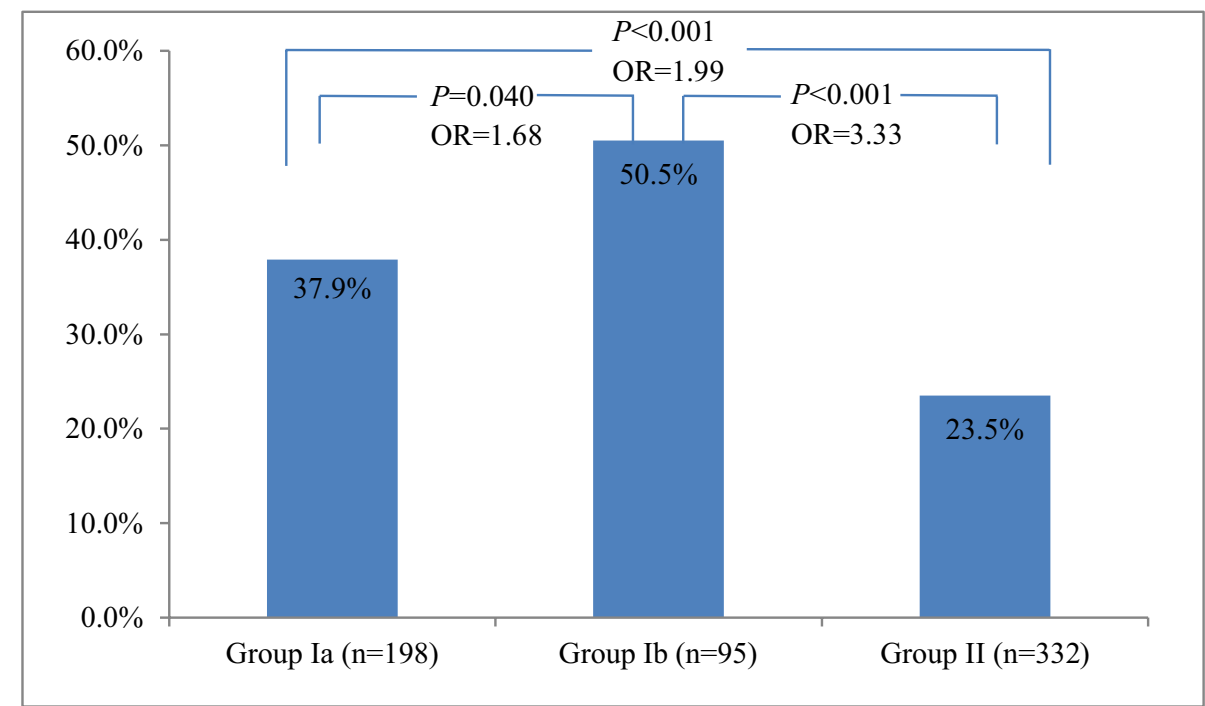

Group Ia-women with a classical history of cervical incompetence

Group Ib-women with atypical history of cervical incompetence

Group II-Women with recurrent first trimester loss (the comparison group)
$55 / 141(39.0 \%)$ and $30 / 82(36.6 \%)$ respectively $(3 \times 2$ contingency table analysis, $P=0.054)$.

Within group II, the prevalence of CE in subjects with 2 first-trimester miscarriages and $\geq 3$ miscarriages were $47 / 205(22.9 \%)$ and $31 / 127(24.4 \%)$ respectively $(2 \times 2$ contingency table analysis, $P=0.757)$.

\section{Factors affecting the prevalence of chronic endometritis in study group}

To analyze possible confounding variables, regression analysis was performed using chronic endometritis as dependent variable and age, BMI, parity, number of mid-trimester loss, number of first trimester loss, typical or atypical mid-trimester as independent variables. The prevalence of chronic endometritis was found to be significantly corelated with the type of CI (typical or atypical, $P=0.034$ ), but not with age, BMI, parity, number of first trimester loss and number of mid-trimester loss.

\section{Outcomes of subsequent pregnancy after LCC}

Among 293 women in Group I, 247 women underwent preconception LCC, of whom 7 women were lost to follow, 29 women had either delayed period or biochemical loss and 211 women had ultrasound evidence of conception. Among the 211 conceptions confirmed by ultrasound, the first trimester miscarriage rate, mid-trimester loss rate, preterm delivery rate and term delivery rate were 2.8, 1.9, 12.3 and $82.9 \%$ respectively.

The mid-trimester loss rate, preterm delivery rate and term delivery rate were not significantly different (1) between women with and without CE $(2.2,12.0,85.8 \%$ vs. 
$1.8,10.1$ and $88.1 \%$ respectively), and (2) between women with $\mathrm{CE}$ treated and not treated with antibiotics prior to conception $(2.3,9.3,88.4 \%$ vs. $2.0,14.3$ and $83.7 \%$ respectively), excluding 4 cases that underwent preterm delivery as a result of other complications (3 cases of pregnancyinduced hypertension syndrome and 1 case of placenta previa).

In addition, there was no difference in first trimester miscarriage rate $(2.9 \%$ vs. $2.8 \%)$, mid-trimester loss rate $(0.7 \%$ vs. $4.2 \%)$, preterm delivery rate $(12.2 \%$ vs. $12.5 \%)$ and term delivery rate $(84.2 \%$ vs. $80.5 \%)$ between groups Ia and $\mathrm{Ib}$.

The relationship between obstetrics outcome (mid-trimester loss or preterm delivery vs. term delivery) and various clinical parameters was analyzed in Table 3 . There was no significant difference in any of the parameters between the two groups $(P>0.05)$.

\section{Adverse events arising from the cerclage}

Among the subjects who underwent cerclage, there were 2 cases of intra-operative bleeding of more than $100 \mathrm{ml}$ $(0.8 \%), 1$ case of perforation of uterus $(0.4 \%)$, with no case of bladder or ureteric injury, wound or urinary tract infection, inadvertent placement of suture through the cervical canal, adverse reaction to antibiotic therapy and none required conversion to laparotomy.

\section{Discussion}

This retrospective cohort study aimed to investigate the prevalence of chronic endometritis (CE) among women with cervical incompetence $(\mathrm{CI})$ as a main contributor for midtrimester loss. We have found that women (group I, 42.0\%) with mid-trimester loss associated with cervical incompetence $(\mathrm{CI})$ appeared to have an increased prevalence of $\mathrm{CE}$ compared to women (group II, 23.5\%) with recurrent early miscarriage but without second trimester loss. Some studies $[18,19]$ reported that $\mathrm{CE}$ was associated with unexplained recurrent first trimester miscarriage, but our study suggests that the association appeared to be even stronger with midtrimester loss due to CI than recurrent early miscarriage. Monsanto et al. [20] concluded that cervical insufficiency was associated with local inflammation in cervicovaginal fluid.

However, it is uncertain if the association between $\mathrm{CE}$ and spontaneous mid-trimester loss due to $\mathrm{CI}$ is casual or causal. It is uncertain if pre-existing $\mathrm{CE}$, which is often asymptomatic, may lead to sub-clinical increase in uterine activity in the second trimester which over time causes the cervix to shorten and open up. On the other hand, it is possible that the eventual presentation of CI depends on whether or not there is coexistence of CE. In our study, the prevalence of $\mathrm{CE}$ in women presented with an atypical
Table 3 A comparison of the demographic and clinical details between women who had (1) mid-trimester loss or preterm delivery and (2) term delivery

\begin{tabular}{llll}
\hline & $\begin{array}{l}\text { Mid-trimester loss or preterm } \\
\text { delivery }(n=26)\end{array}$ & Term delivery $(n=175)$ & $P$ value \\
\hline Age*,$(\text { year })^{2}$ & $33(21-43)$ & $32(23-42)$ & 0.524 \\
BMI*, $\left(\mathrm{kg} / \mathrm{m}^{2}\right)$ & $23.4(18.4-37.8)$ & $22.7(18.7-38.5)$ & 0.438 \\
Parity, $n=$ & & 0.137 \\
0 & 26 & 157 & \\
$\geq 1$ & 0 & 18 & 0.823 \\
Previous mid-trimester loss, $n=$ & & \\
1 & 5 & 37 & 0.441 \\
$\geq 2$ & 21 & 138 & \\
Previous first trimester loss, $n=$ & & \\
1 & 23 & 162 & \\
$\geq 2$ & 3 & 13 & \\
Cervical incompetence type & & \\
Typical & 16 & 113 & 0.763 \\
Atypical & 10 & 62 & \\
CE type & & & \\
Negative & 13 & 96 & \\
Positive & 13 & 79 & \\
Treatment with CE & & 38 & \\
No & 8 & & \\
Yes & 5 & & \\
\hline
\end{tabular}

*Median (range in brackets); CE, Chronic endometritis 
history of CI (50.5\%) was significantly higher than that of women presented with a classical history of CI (37.9\%). This indicates women with a typical history of $\mathrm{CI}$ (painless dilatation of cervix) is less likely to have coexisting CE compared to women with an atypical history.

In women with CI, there may be asymptomatic dilatation of the cervix for some time before the women presented with bulging membranes and expulsion of the fetus (typical cases). During the latent phase of cervical dilatation, however, secondary ascending infection may occur, leading to a variable degree of uterine contraction. In this situation, the presentation may be somewhat atypical, including uterine tightening, backache and rarely strong uterine contractions and ruptured membranes. Following ruptured membranes, the pressure of the fore-water on the cervix is reduced, the degree of cervical dilatation may regress, and cervical dilatation may no longer be as obvious after the membranes have ruptured. The presentation of $\mathrm{CI}$ is therefore dependent on the coexistence of any secondary infection. It may also explain why sometimes histopathological examination of the placenta in genuine cases of CI showed evidence of chorion-amnionitis, and why the presence of the latter should not be used as evidence against a diagnosis of CI. It is important to appreciate that the two contributory factors for mid-trimester loss, namely CI and infection, may coexist and together influence the presentation.

In an earlier study, antibiotic therapy in women with chronic endometritis was reported to increase clinical pregnancy rate and live birth rate [12]. In our cohort study, we observed that the term delivery rate of women who did not receive antibiotic therapy for CE (83.7\%) was slightly lower but not significantly different to those who did receive antibiotic therapy $(88.4 \%)$. Nevertheless, the observation should be considered preliminary for a number of reasons, namely, the sample size was not sufficiently powered to address the impact of antibiotic therapy, the retrospective nature of the study makes it more prone to bias and there are confounding variables including the possibility that some subjects may have received prophylactic antibiotic therapy around the time of cerclage or additional courses of antibiotic as guided by the result of high vaginal swab which are more often performed in this group of women.

A limitation of our study was the retrospective nature of the study and the inclusion of various subsets of population; our observation should be used to guide the planning of a prospective study with defined subsets of women with midtrimester loss to confirm the findings.

The relationship between alteration of cervical microbiome and recurrent miscarriage or preterm labor has recently been examined [21, 22]. In this study, we have examined the relationship between mid-trimester loss and CE based on histological evaluation of the endometrium. However, molecular microbiological study of the genital tract and maternal-fetal interface, a rapidly developing area, may throw further light on the relationship between mid-trimester loss, altered microbiome and specific viral infection such as Covid-19. Our observations lend support to the need to conduct future studies on the relationship between microbiome in endometrial cavity and mid-trimester loss and preterm labor. It remains to be seen if microbiome or its alteration in the endometrial cavity plays an important role in midtrimester loss and preterm labor.

The current study highlighted that CE should be added to the list of factors including genetic, structural (uterus and cervix), infection and environmental (such as tobacco) which ought to be considered in women at risk of mid-trimester loss or preterm delivery.

\section{Conclusion}

The prevalence of CE in women with mid-trimester loss associated to CI was significantly higher than that of women presented with recurrent first-trimester loss. Women with a typical history of CI (painless dilatation of cervix) is less likely to have coexisting CE compared to women with an atypical history. However, the presence or not of CE and whether it was treated with antibiotics prior to conception did not appear to significantly influence the obstetric outcomes of women with CI after LCC.

Supplementary Information The online version contains supplementary material available at https://doi.org/10.1007/s00404-021-06029-3.

Acknowledgements We express our gratitude to Yu Xiao, Qiaoyun Zhou, Yan Guo for their assistance during this study.

Author contributions JL Project development, Data collection, Data analyses, Manuscript writing. DS: Project development, Data collection. XF: Data collection, Laboratory supervision. XH: Clinical supervision, manuscript revision. EX: Clinical supervision. TCL: Project development, Data analyses, Manuscript writing.

Funding None.

\section{Declarations}

Conflict of interest The authors declare that they have no conflicts of interest and nothing to disclose.

Ethics approval Ethics approval from Fu Xing Hospital, Capital Medical University IRB (Approval Notice Number: 2015FXHEC-KY005) was obtained in April 2015.

Informed consent Informed consent was obtained from all individual participants included in the study. 


\section{References}

1. Drakeley AJ, Quenby S, Farquharson RG (1998) Mid-trimester loss-appraisal of a screening protocol. Hum Reprod 13(7):1975-1980

2. El HH, Crepaux V, May-Panloup P, Descamps P, Legendre G, Bouet PE (2017) Recurrent pregnancy loss: current perspectives. Int J Womens Health 9:331-345

3. Srinivas SK, Ma Y, Sammel MD et al (2006) Placental inflammation and viral infection are implicated in second trimester pregnancy loss. Am J Obstet Gynecol 195(3):797-802

4. Mastrolia SA, Baumfeld Y, Hershkovitz R, Yohay D, Trojano G, Weintraub AY (2018) Independent association between uterine malformations and cervical insufficiency: a retrospective population-based cohort study. Arch Gynecol Obstet 297(4):919-926

5. Pandey S, Murdia K, Chandra V, Murdia A (2018) Re: Genetic variation in the progesterone receptor gene and susceptibility to recurrent pregnancy loss: a case-control study: Progesterone receptor genetic variants as predictors of recurrent pregnancy loss: an epidemiological study. BJOG 125(6):761

6. Pandey S, Murdia K, Murdia A, Chandra V, Murdia N (2018) Re: Partner smoking influences whether mothers quit smoking during pregnancy: a prospective cohort study. BJOG 125(7):904

7. Pandey S, Tyagi R (2014) Risk factors for miscarriage from a prevention perspective: a nationwide follow-up study. BJOG 121(11): 1439

8. Pandey S, Murdia K, Murdia A (2017) Tobacco as a significant predictor of infertility: a public health perspective in an Indian scenario. Fertil Sci Res 4(1):15-21

9. Sneider K, Christiansen OB, Sundtoft IB, Langhoff-Roos J (2017) Recurrence rates after abdominal and vaginal cerclages in women with cervical insufficiency: a validated cohort study. Arch Gynecol Obstet 295(4):859-866

10. Kannar V, Lingaiah HK, Sunita V (2012) Evaluation of endometrium for chronic endometritis by using syndecan-1 in abnormal uterine bleeding. J Lab Physicians 4(2):69-73

11. Kitaya K, Yasuo T (2011) Immunohistochemistrical and clinicopathological characterization of chronic endometritis. Am J Reprod Immunol 66(5):410-415

12. Johnston-MacAnanny EB, Hartnett J, Engmann LL, Nulsen JC, Sanders MM, Benadiva CA (2010) Chronic endometritis is a frequent finding in women with recurrent implantation failure after in vitro fertilization. Fertil Steril 93(2):437-441
13. Bouet PE, El HH, Monceau E, Gariépy G, Kadoch IJ, Sylvestre C (2016) Chronic endometritis in women with recurrent pregnancy loss and recurrent implantation failure: prevalence and role of office hysteroscopy and immunohistochemistry in diagnosis. Fertil Steril 105(1):106-110

14. McQueen DB, Perfetto CO, Hazard FK, Lathi RB (2015) Pregnancy outcomes in women with chronic endometritis and recurrent pregnancy loss. Fertil Steril 104(4):927-931

15. ACOG Practice Bulletin No.142 (2014) Cerclage for the management of cervical insufficiency. Obstet Gynecol 123(2 Pt 1):372-379

16. Song D, Feng X, Zhang Q et al (2018) Prevalence and confounders of chronic endometritis in premenopausal women with abnormal bleeding or reproductive failure. Reprod Biomed Online 36(1):78-83

17. Huang X, Ma N, Li TC et al (2016) Simplified laparoscopic cervical cerclage after failure of vaginal suture: technique and results of a consecutive series of 100 cases. Eur J Obstet Gynecol Reprod Biol 201:146-150

18. Zolghadri J, Momtahan M, Aminian K, Ghaffarpasand F, Tavana $\mathrm{Z}$ (2011) The value of hysteroscopy in diagnosis of chronic endometritis in patients with unexplained recurrent spontaneous abortion. Eur J Obstet Gynecol Reprod Biol 155(2):217-220

19. Kitaya K (2011) Prevalence of chronic endometritis in recurrent miscarriages. Fertil Steril 95(3):1156-1158

20. Monsanto SP, Daher S, Ono E et al (2017) Cervical cerclage placement decreases local levels of proinflammatory cytokines in patients with cervical insufficiency. Am J Obstet Gynecol 217(4):455.e1-455.e8

21. Sebire NJ (2001) Choriodecidual inflammatory syndrome (CoDIS) is the leading, and under recognised, cause of early preterm delivery and second trimester miscarriage. Med Hypotheses 56(4):497-500

22. Nigro G, Mazzocco M, Mattia E, Di RGC, Carta G, Anceschi MM (2011) Role of the infections in recurrent spontaneous abortion. $\mathrm{J}$ Matern Fetal Neonatal Med 24(8):983-989

Publisher's Note Springer Nature remains neutral with regard to jurisdictional claims in published maps and institutional affiliations. 